\title{
Biosensor of Urine Analysis Based on Graphene Nano Sheet
}

\author{
Ashraf Abdel Raheem ${ }^{1 *}$, Ashraf Mahroos², Mohamed Salah¹, Ibrahim Ashour ${ }^{1,3}$ \\ ${ }^{1}$ Department of Chemical Engineering, Faculty of Engineering, Minia University, El-Minya, Egypt. \\ 2 Department of Biomedical Engineering, Faculty of Engineering, Minia, University, El-Minya, Egypt. \\ 3 Environmental Engineering, Zewail City of Science and Technology, 6th October, Egypt. \\ * Corresponding author. Tel.: 00201004870136; email: Ashraf.engone2010@gmail.com \\ Manuscript submitted March 9, 2017; accepted August 25, 2017. \\ doi: 10.17706/ijbbb.2018.8.1.53-60
}

\begin{abstract}
The aim of this work to propose a stable and easy technique to analyze urine based on graphene Nano sheet. Where graphene Nano sheets used as a detector and as well as the rate of electrons transfer into the detector. As a result, the characteristic surface conductivity of graphene makes it a super conductor in a very small size. Urea measurement in urine and blood related to many diseases such as kidney and liver diseases. Sensitive urea measurement in urine and blood really need super conductor graphene sensor which can read the concentration of urea and translate it to micro and milli ampere at specific voltage then compare them with a normal one. The graphene super conductor material is chemical inert which can read a very low concentration and make an accurate prediction of theses serious diseases without any effect on the results within a simple, it produces a stable and accurate performance.
\end{abstract}

Key words: Grapheme, urea, nano sheet, biosensor and super conductor.

\section{Introduction}

Urea, a main end product of protein metabolism, can act as an important indicator of liver and kidney function. A normal range is about 12 to 20 grams/24 hours, depending on the build and relative health of the body. A decreased urea level can be associated with severe liver disease or insufficient protein intake. A high urea concentration can cause renal dysfunction, urinary tract obstruction, dehydration, shock and gastrointestinal bleeding. Therefore, it is very important to monitor the level of urea to determine the health of the livers and kidneys in the human body. Since many decades, some analytical methods have been developed for urea detection, such as enzymatic assay, gas chromatography, calorimetry, high-performance liquid chromatography), fluorimetry, surface plasmon resonance and electrochemistry, Despite many advances in urea detection, many of these methods still do not meet the growing demand for more sensitive and selective detection of urea. Thus, the further development of simple, facile and stable methods with high sensitivity and selectivity for urea detection is highly needed [1].

The ultra-high carrier mobility of graphene and its theoretical specific surface area is as high as $2630 \mathrm{~m}^{2}$ $\mathrm{g}^{-1}$ gives a promising potential for the fast operating speed of graphene-based transistors [2]. In addition, the peak intrinsic average carrier velocity of graphene was theoretically calculated to be four time higher than Si. Therefore, graphene is suitable to be used in high-frequency devices, which is of great importance in the application of communication technologies such as wireless transmission and signal processing. The research group from IBM demonstrated the first experimental study on high-frequency top-gated devices 
made of graphene transistors. A high cutoff frequency of $26 \mathrm{GHz}$ was obtained with a channel length of 150 $\mathrm{nm}$. Later, the IBM research group further demonstrated that the cutoff frequency could be increased to 100 $\mathrm{GHz}$ with a gate length of $240 \mathrm{~nm}$ when using graphene grown on Sic. Graphene is currently being high research in the application of the lithium ion (Li-ion) battery, which is a renewable and environmental power source for portable devices, electrical vehicles, and for many power devices. As many potential electrode materials (graphite or transition metal oxide) in a Li-ion battery suffer from slow Li-ion diffusion, poor electron transport, and increased resistance at high charge-discharge rates, graphene-based electrode materials in Li-ion batteries have been proposed as one of the promising alternatives due to graphene's high electrical conductivity and typical 2-D structure . Nowadays many researchers have been using graphene as a high-capacity anode material. More importantly, graphene is acting as a conducting agent with unique 2-D nanostructure and it is helpful for fabrication of novel structures with various active materials and enormously improving their carrier mobility performance. So far, numerous graphene-based composite cathode and anode materials have been successfully prepared, and their outstanding charge/discharge performance shows the broad future of graphene in the application in Li-ion batteries [3]. Graphene based biosensors were good studied because of the large surface area and good electrical, mechanical, thermal, and biocompatibility properties of graphene [4]. Biosensors need electrical conductivity as well as good adsorption of biomolecules to induce strong substrate-molecule coupling. Metallic nanoparticles, mostly used in biosensors, possess excellent electrical conductivity, but have poor biomolecules absorption. Graphene is a very attractive option due to its excellent electrical conductivity along with its special molecular structure, which favors biomolecule adsorption through $\pi$-stacking interactions [5]. Intrinsically conductive polymers (CPs) have been intensively studied in recent years due to potential use in several applications such as electronic, and photo electrochemical devices; rechargeable batteries, smart windows, sensors, light-emitting diodes;8 and non-linear optical devices [6]. SO, the widely development of graphene research leaves emphasis that this material will revolutionize several markets like electronics, medical application, and energy storing in the near future. All these important innovations, which were generated after the first isolation of graphene layers, indicate that the use of these materials is not limited to providing simply a theoretical model that can describe the physical properties of several organic nanostructures. Graphene is occupying a centerpiece position in many scientific advances that can change our way of making and using technology [5].

Many researches in bio sensing and the enhancement of grapheme performance reported that, the addition of nitrogen to graphene has shown significant promise in the improvement of biocompatibility of carbon devices [7]. Synthesis method to prepare $\mathrm{Fe}_{2} \mathrm{O}_{3}$ /graphene composite anode material by homogeneous precipitation of $\mathrm{FeCl}_{3}$ in the graphene oxide (GO) suspension with urea and subsequent chemical reduction of GO into graphene under microwave irradiation [3], [8]. Electrochemical reduction of exfoliated GO in an acetamide-urea-ammonium nitrate ternary eutectic melt resulted in few-layer graphene thin films [9]. Urea has been used as an environmentally friendly reductant to prepare grapheme because the decomposition of urea not only creates volatile species that mechanically expand GO, but also produces volatile reducing gases that can promote the removal of surface oxygen groups [3].

\section{Experimental Work}

\subsection{Materials and Apparatus}

Urea has been used as an environmentally friendly reductant to prepare graphene [10]. Here now graphene will be used as detector or biosensor to determine urea concentration depending on its high conductivity and ultra-high carrier mobility and also we investigate electrical activity of different urine concentrations. On the other hand, its excellent chemical stability, high electrical conductivity, flexible and 
large surface area makes it attractive in biosensor [11]. Graphene is the best electrical conductor of any known metal, high electron mobility (at room temperature $\sim 200.000 \mathrm{~cm}^{2} /(\mathrm{v} \bullet \mathrm{s}$ ), ex. Si at RT 1400 $\mathrm{cm}^{2} /(\mathrm{v} \bullet \mathrm{s})$, carbon nanotube: $\sim 100.000 \mathrm{~cm}^{2} /(\mathrm{v} \bullet \mathrm{s})$.

$$
v \mathrm{~d}=\mu \mathrm{E}
$$

where $u d$ is the drift velocity in $\mathrm{m} / \mathrm{s}$, (SI units), E is the applied electric field in $V / \mathrm{m},(\mathrm{SI})$ and $\mu$ is the mobility in $\mathrm{m}^{2} /(\mathrm{v} \bullet \mathrm{s})$, in (SI).

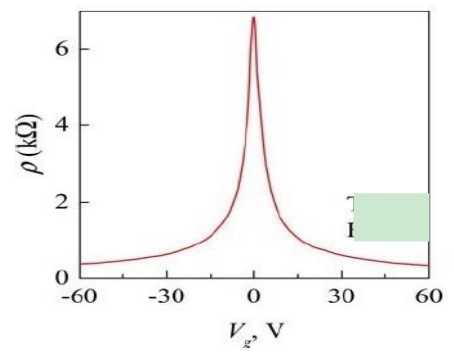

Fig. 1. (a) Resistivity $\rho$ of few-layer graphene on gate voltage (Vg) [12].

Fig. 1 indicates ambipolar electric field when it applies in a single layer of graphene. The rapid decrease in resistivity under applying the electric field proves the high mobility of graphene. So this large surface area, high electrical conductivities and low noise response makes graphene a good sensor. Small change in carrier concentration induced by material exposure could lead to significant changes in electrical conductivity. In other words, materials analytes were detected by measuring the resistance changes of sensing layers induced by concentration the molecules, good electrical properties to changes in the surroundings [13].

Graphene based sensors work on the principle that interaction of material molecules changes the local charge carrier mobility in graphene by either increasing or decreasing the concentration of electrons depending on the nature of material species (electron donor or acceptor) which leads to corresponding decrease or increase in electrical conductivity [11]. In the traditional methods when use graphene as conductor by using substrate. But when graphene is used with substrate, the interaction between $\pi$ electrons of graphene and the substrate electrons makes change of the electronic structure and lower the carrier mobility of graphene. For example, when we use $\mathrm{SiO}^{2}$ as the substrate with graphene, the carrier mobility of graphene is limited to $\sim 4 \times 104 \mathrm{~cm}^{2} \mathrm{~V}^{-1} \mathrm{~s}^{-1}$ at room temperature. In addition, interactions between $\pi$ electrons with the substrate are main responsible for the presence of many impurity scattering in graphene, which restricts the movement of electrons and thus reduce carrier mobility [14], [15]. So we used carbon cross as a substrate and made graphene layer stick with carbon cross surface with solution of Nafion ethanol technique (10 $\mathrm{mm}$ of ethanol and $5 \mathrm{~mm}$ of Nafion solution) mixed with graphene to be ready for sensor fabrication.

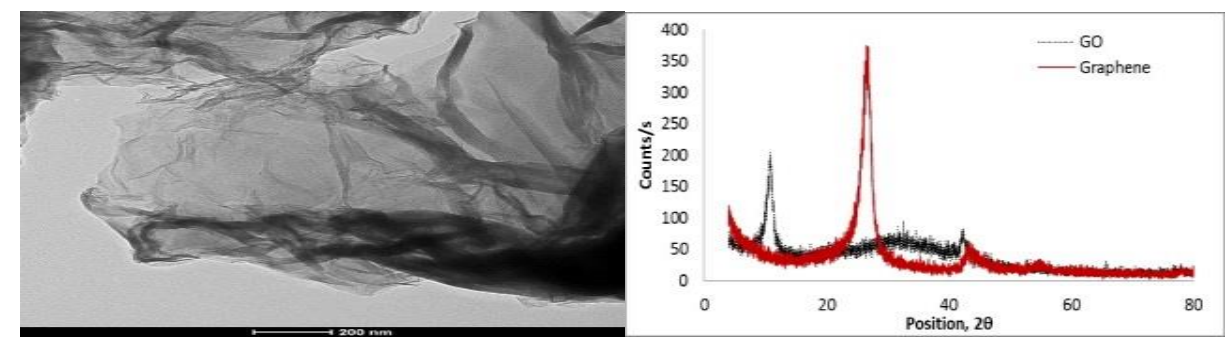

Fig, 2. (a) SEM image of graphene nano sheet (b) XRD pattern of the graphene/graphene oxide. 
Fig. 2 (a) and (b) shows SEM images and XRD pattern of graphene which we used it for fabrication of graphene Nano sheet as biosensor. Graphene sheet was fabricated by deposing graphene solution on carbon cross sheet and dry in the furnace at $95 \mathrm{C}^{0}$ for two hours several times.

Graphene for highly conductive transparent thin films was prepared by electrochemical exfoliation of graphite as shown in the block diagram, and grapheme Nano sheet biosensor is immersed in urine sample and then it is connected electrically to Camry instrument to infer and detect (Fig. 3).

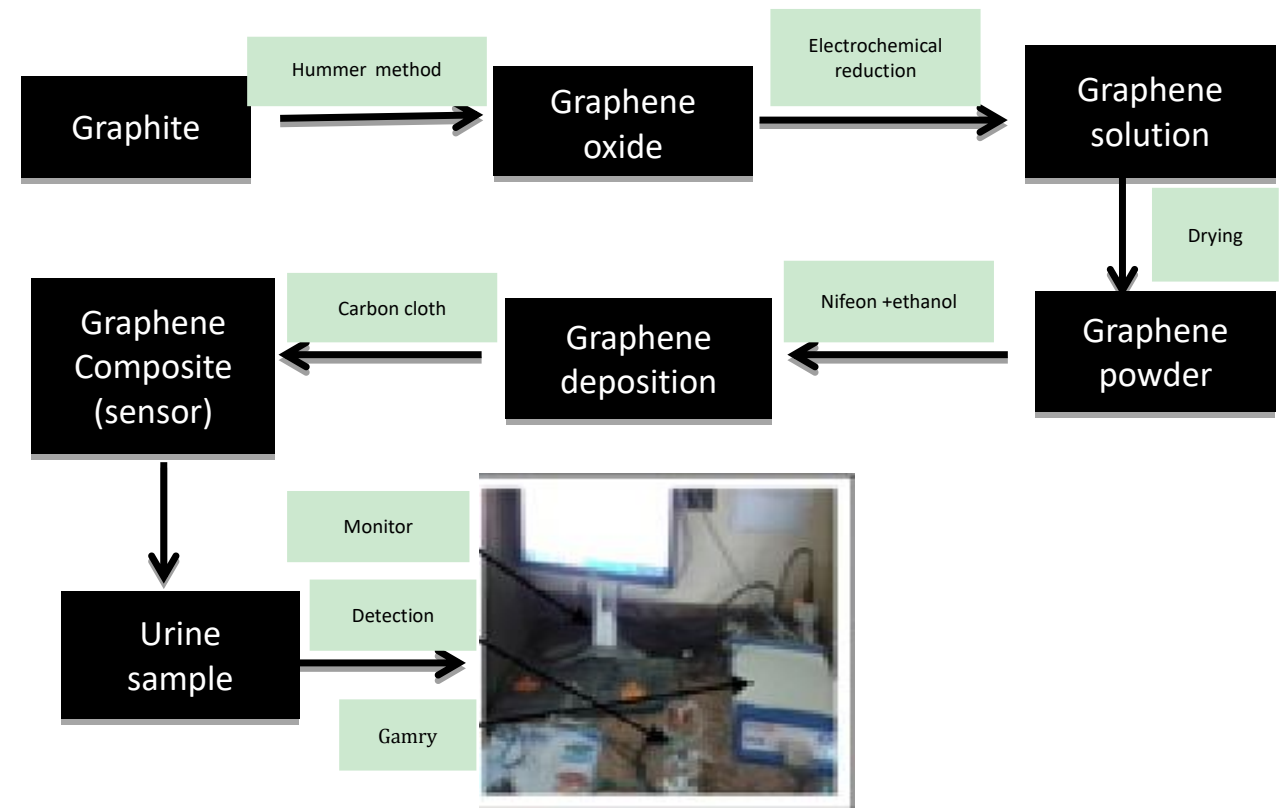

Fig. 3. Block diagram of graphene preparation and biosensor system.

\subsection{Results and Discussion}

Gamry Instrument that make detection for deferent urine samples, male, female and kids and draw graphs by connecting with computer as demonstrated shown in figures and compare them with normal curve. In this work we used the same samples which used in traditional analysis by titration methods. Theses Routine methods didn't detect any different of urea concentration in urine samples. But by grapheme sensor the result indicate any increase or decrease of urea at any concentration.

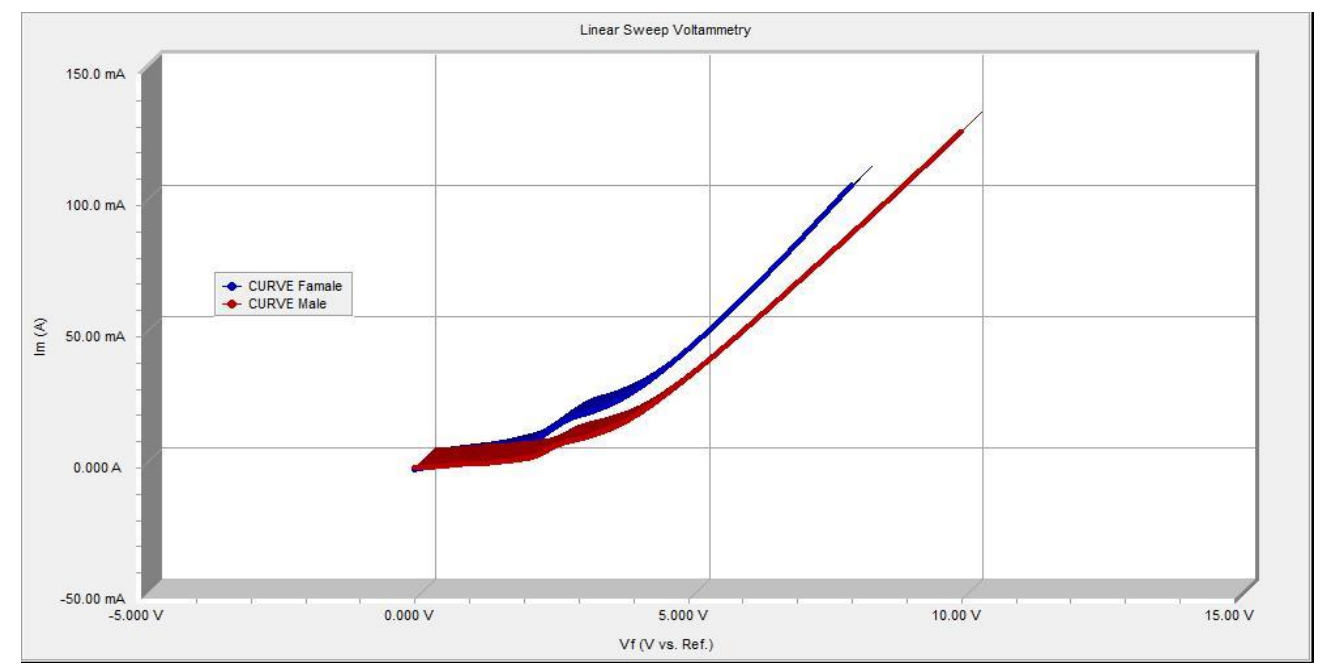

Fig. 4. Graphene biosensor detect, for male and female samples. 
Fig. 4 reveals different concentration of urea for male and female samples. The conventional analysis tells us that both have urine. So the same result and the same doses of medicine must be used. But with our detector which shows that the female has higher concentration than male so must have more doses.

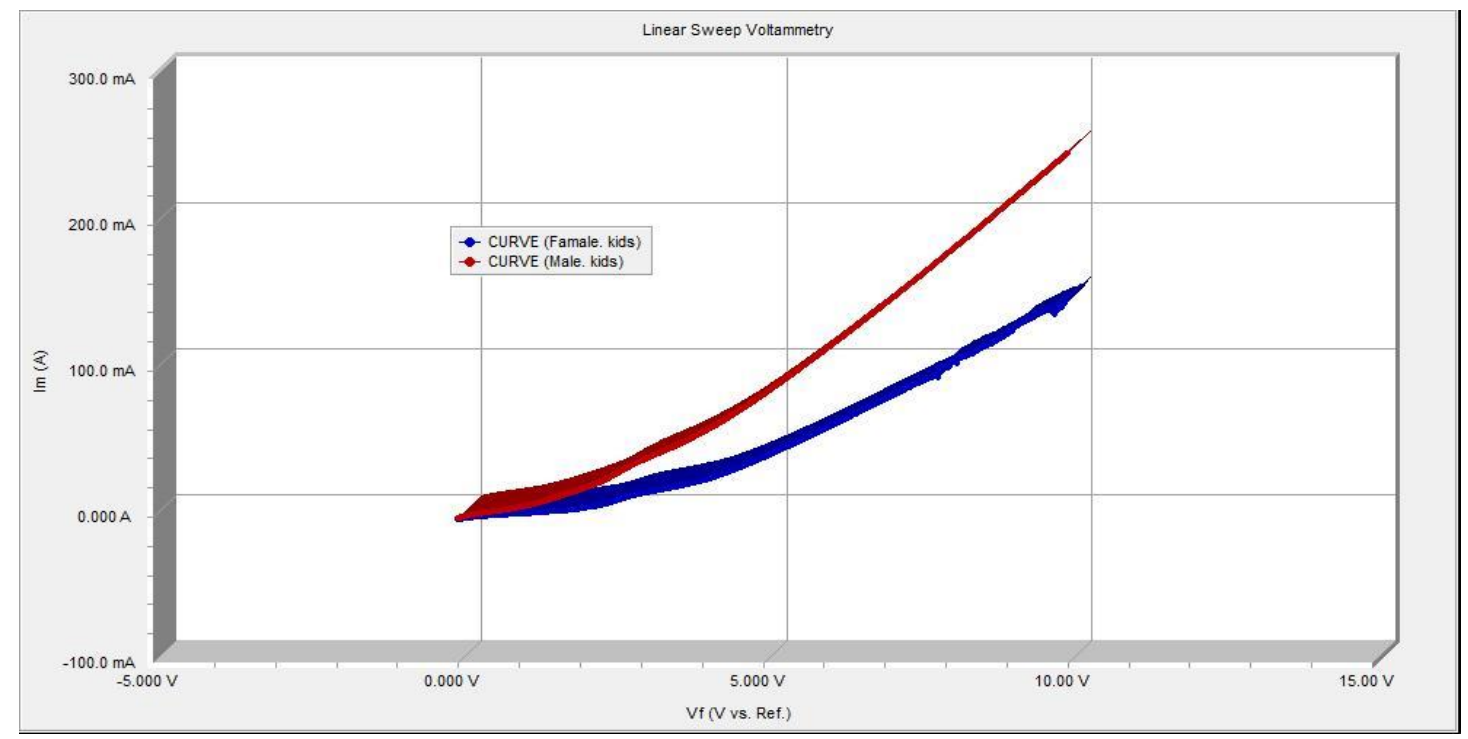

Fig. 5. Graphene biosensor detects, for male and female kids samples.

Fig. 5 demonstrates the analysis for two children also have the same analysis. But the high defrent is very clear. The boy has higher than the girl. So the boy must have doses of medicine than the girl.

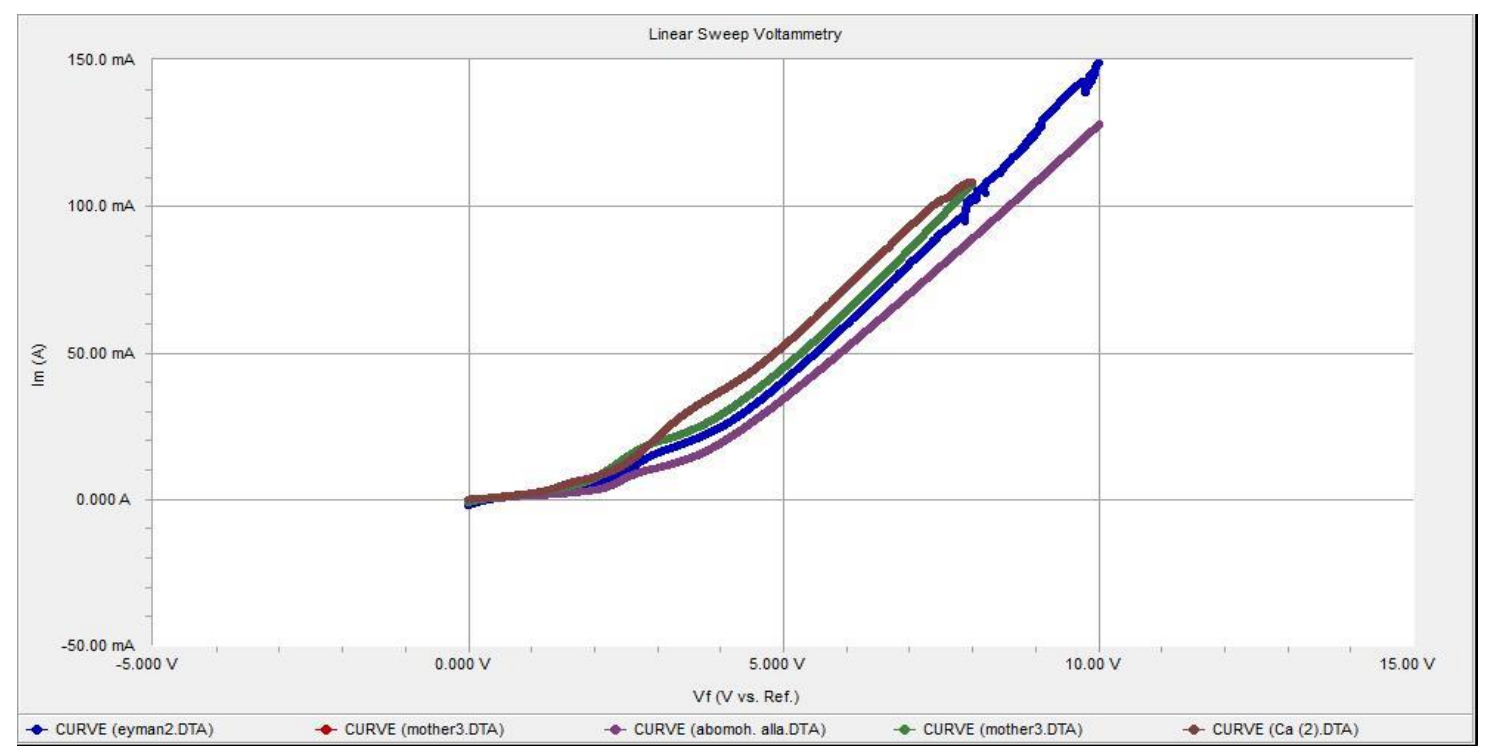

Fig. 6. Different urea concentration at the same condition.

Fig. 6 depicts the analysis for family. The first look tells us the same conditions, eating and drinking due to the same results. But our detector tells us anther behavior. The mother and her daughter nearly the same analysis but the father and his son have a wide range urea concentration.

Fig. 7 shows current stability after 10 seconds which we can take the maximum current for different urine concentration. Stability of maximum current can be the based for fabricating the sensor design. This figure emphasis the results in the other curves that high concentration urea has high concentration current. 


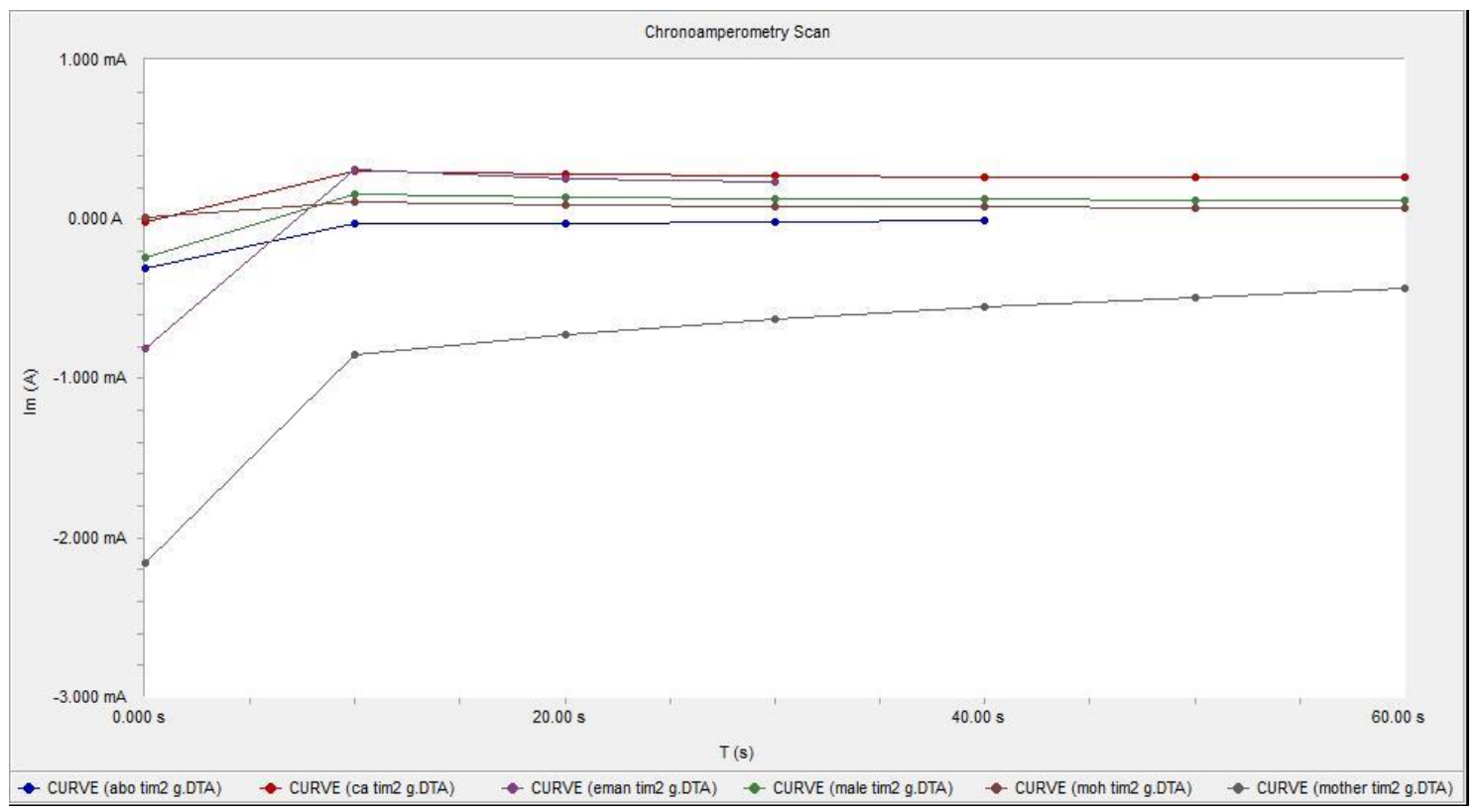

Fig. 7. Current stability after 10 seconds.

\section{Conclusion and Recommendation for Future Work}

The graphene biosensor is a start of super conductor. It is chemical inert flexible, high conductivity, cheap, alternative to silicon and metal based electronics the European Union is spending 1.2 billion dollars on research into graphene. In this research make and development urine detector as we show it can detect with very accurate increase or decrease in urea concentration which related to serious diseases such as kidney and liver. By simple detector and easy use we can determine the urea concentration due to eating. Drinking or diseases .we will develop this detector to detect urea in blood and to be handling for any one.

\section{Acknowledgment}

The authors gratefully acknowledge support of this work by for providing technical support to the team.

\section{References}

[1] Yang, Z.-P., et al. (2015). A high-performance nonenzymatic piezoelectric sensor based on molecularly imprinted transparent tio2 film for detection of urea. Biosensors and Bioelectronics, 74, 85-90.

[2] Chen, C.-M. (2016). Surface Chemistry and Macroscopic Assembly of Graphene for Application in Energy Storage. Springer-Verlag Berlin Heidelberg.

[3] Liu, Z. P., \& Zhou, X. F. (2015). Graphene Energy Storage and Conversion Applications. CRC Press Taylor \& Francis Group.

[4] Sekhar, C. R. (2015). Applications of Graphene and Graphene-Oxide Based Nanomaterials. Elsevier Inc.

[5] Choi, W. B., \& Lee, J. W. (2112). Graphene Synthesis and Applications. Taylor \& Francis Group, LLC.

[6] Vikas, M. (2012). Polymer-Graphene Nanocomposites. The Royal Society of Chemistry, 223.

[7] Rashid, M. Y. (2015). Graphene-Based Energy Devices. Wiley-VCH.

[8] D'Souza, F., \& Karl, M. K. (2014). Graphene - Fundamental Properties, 5, 321.

[9] Viera, S., \& Alan, B. K. (2014). Graphene Properties, preparation, characterization and devices. Woodhead Publishing Series in Electronic and Optical Materials, 57.

[10] Rao, C. N. R., \& Sood, A. K. (2013). Graphene Synthesis, Properties, and Phenomena. Wiley-VCH Verlag GmbH \& Co. KGaA. 
[11] Ashutosh, T., \& Mikael, S. (2015). Graphene Materials _ Fundamentals and Emerging Applications Scrivener Publishing.

[12] Amin, K. R., \& Bid, A. (2014). Graphene as a sensor. Current Science, 107(3), 431.

[13] Igor, L. S. (2014). Ultra-High Temperature Materials I_ Carbon (Graphene_Graphite) and Refractory Metals. Springer Netherlands.

[14] D’Souza, F., \& Karl, M. K. (2014). Graphene Fundamental Properties, 5, 321.

[15] Sara, V., et al. (2015). Two-dimensional materials for sensing: Graphene and beyond. Electronics, 4, 651-687.

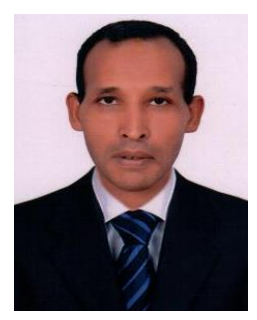

Ashraf Abdel Raheem graduated from chemical engineering in Mania University, his master degree, M.Sc. in oleo chemicals industry (mono, di and triglycerides production from waste free fatty acids) in 2013. He has certified operation manger in Almnaar Company 2014. He worked in RO water treatment operation and maintenance. He is currently studding $\mathrm{PhD}$ graduate in Mania University. His scope of research interests in Biomedical engineering and chemical engineering in Nano technology research.

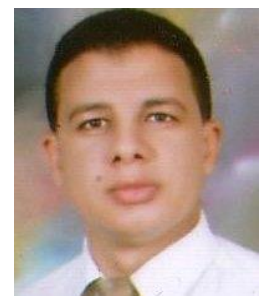

Ashraf M. Said graduated from biomedical engineering in Cairo university, his master degree, M.Sc. in optimum design of MRI gradient and RF coils in 2005 while Ph.D. is the statistical analysis of Brian connectivity using Bold images of FMRI on human vision system, the Ph.D. graduation in 2011, He is currently an Assistant Professor in Biomedical Engineering department in Faculty of engineering in Minia University. His scope of research interests in Bio signal processing, recognition in biometric, Image processing, biotechnology, and biomedical application of nanotechnology

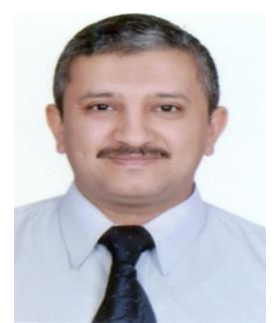

Mohamed S. Mahmoud was born in Kuwait on 7/11/1976. He got his BSc from Chemical Engineering Department, Faculty of Engineering, Minia University, Egypt at 1999, and the MSc degree from Chemical Engineering Department, Faculty of Engineering, Minia University in 2003. The field of study was fluid mechanics, then he got his PhD from Tokyo Institute of Technology, Japan in 2008 in the field of renewable energy.

He works as associate professor at Department of Chemical Engineering, Faculty of Engineering, Minia University. He has experience in laser ablation for metal oxide reduction, physical vapor deposition under vacuum and inert gas conditions, water desalination by humidification dehumidification technique, photocatalytic water splitting, graphene synthesis and application as catalyst for ethanol fuel cell, and application of magnetic field for wastewater treatment. He published 22 papers in the previous mentioned fields, also, he attended 15 international conference in the specified fields. Moreover, he worked as visiting researcher at Tokyo institute of technology from 2014-2015.

Dr Mohamed is a member of Egyptian syndicate of engineers, reviewer for desalination and water treatment journal. He participated as Co PI in three research project, also, He was the contact PI of one project awarded by research development and innovation program with the cooperation of European Union. He was awarded the Egypt State incentive prize for engineering science in 2015.

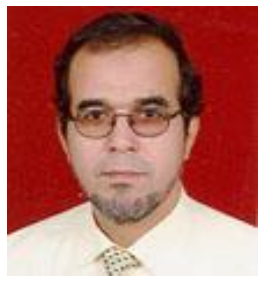

Ashour earned his B.Sc. in chemical engineering in 1977 from Cairo University with distinction. He earned his M.Sc. in 1983 from Cairo University and Ph.D. in 1989 from Lund University at Lund, Sweden with a major in chemical engineering and specialty in applied thermodynamics and separation. Prof. Ashour worked in several funded projects in supercritical fluid extraction, extraction of essential oils and Fatty acids fractionations 
and glycerol water separation. Before joining BUE in November 2011, Prof. Ashour was head of the Department of Petroleum and Chemical Engineering at Sultan Qaboos University, Muscat, Oman. He had been working in many places, 1990-1995 Elminia University, Egypt, 1995-1997 KFUPM, Saudi Arabia, 1998-2005 United Arab Emirates University, UAE, 2005- 2011 Sultan Qaboos University (SQU), Oman, 2011- 2014 British University in Egypt, 2014- Present Zewailcity of Science and Technology and Minia University. Prof. Ashour has been working in many areas of research as listed Green Engineering, Applied Thermodynamic and Separation Processes, Natural Gas, , Microbial Fuel Cell, Engineering Education research (Problem solving learning approach in Thermodynamics, comparative study between global curriculum and sequential curriculum), Development of curricula in British University, Minia University, Sultan Qaboos University, Abu Dhabi Universityand Zewail City University of Science and Technology. In all development of the curricula, an integrated technique has been used. I mean divided the program into strings each one input and output, objectives and outcomes for each string have been specified. 